\title{
Clinical Pathology Case Conference
}

\section{Emergency Physician Stress and Morbidity}

\author{
LESLIE ZUN MD, ${ }^{*}$ MICHAEL KOBERNICK, MD† \\ DAVID S. HOWES, MD $\ddagger$
}

\section{CASE PRESENTATION}

Dr David S. Howes: The patient is a 62-year-old woman with a chief complaint of fainting. She had been well until one week prior to admission, when she noted rhinorrhea, occasional fever with chills, myalgias, and decreased vigor. She had a history of mild emphysema and her chronic, minimally productive cough had increased slightly and the sputum was green in color.

Over the same time period, she noted paroxysms of coughing, which left her feeling faint and from which she recovered with 5 to 10 minutes of rest. She experienced occasional substernal chest burning, which she associated with coughing.

The day of admission, the patient climbed two flights of stairs, had a prolonged coughing episode and voluntarily sat down on the landing to keep from fainting and falling. Her husband volunteered that she looked ashen and sweaty, her color improving with approximately 10 minutes of rest.

There was no loss of consciousness, nausea, vomiting, or chest or abdominal pain associated with the episode. No focal neurologic deficit or vertigo was described. She had no prior cardiovascular disease, al-

"Associate of Clinical Medicine, Department of Medicine, Section of Emergency Medicine, Northwestern University Medical School, Chicago, Illinois.

†Attending Physician, William Beaumont Hospital, Royal Oaks Michigan, and Clinical Instructor, Section of Emergency Medicine, University of Michigan Medical School, Ann Arbor.

fEducation Director, Division of Emergency Medicine, Mercy Hospital and Medical Center; Assistant Professor of Clinical Emergency Medicine, University of Illinois College of Medicine, Chicago.

Manuscript received and accepted December 8, 1987

Address reprint requests to Dr Zun: Northwestern Memorial Hospital, Department of Emergency Medicine, 233 E Superior St, Chicago, IL 60611.

Key Words: Stress, physician impairment, ED administration, psychological adaptations.

C1988 by W.B. Saunders Company

0735-6757/88/0604-0014 \$5.00/0 lergies, or medication usage. She denied diabetes mellitus. She had smoked cigarettes for 40 years.

The physical examination revealed an alert, pleasant, minimally overweight woman in no apparent distress. Vital signs were as follows: blood pressure, 150/ $90 \mathrm{mmHg}$; pulse, 84/minute and regular; respirations, $20 /$ minute; temperature, $98.6^{\circ} \mathrm{F}$ orally. There were no orthostatic changes. The pupils were equal, round, and reactive. The optic discs were sharp, and no hemorrhages or exudates were noted in the fundi. The mucous membranes were not pale. The neck was supple and no carotid bruits or thyroid abnormalities were found.

Ausculation of the chest revealed occasional, scattered rhonchi without rales or wheezes. A II/VI systolic ejection murmur at the apex radiating to the aortic area was found. $S_{1} S_{2}$ were normal. No precordial gallops or rubs were heard. The carotid pulses were normal and there were no bruits. The radial, femoral, and dorsalis pedis pulses wcre full and symmetric. The abdomen was soft and bowel sounds were normoactive. There was no organomegaly. The rectal examination revealed soft brown stool which was negative for occult blood.

The extremities were without edema, cyanosis, or rash. Cranial nerves II through XII, patellar, ankle, and brachioradialis deep tendon reflexes were symmetric. Her sensation and motor strength were felt to be normal for her age. The planter reflexes were downgoing. Romberg test and gait were normal.

Initial laboratory findings were as follows: WBC, 8,000 cells $/ \mathrm{mm}^{3}$; hematocrit, $41 \%$; hemoglobin, 13.0 $\mathrm{gm} / \mathrm{dL}$; platelets, 220,000; blood urea nitrogen (BUN), $10 \mathrm{mg} / \mathrm{dL}$; glucose, $118 \mathrm{mg} / \mathrm{dL}$; sodium, $140 \mathrm{mEq} / \mathrm{L}$; potassium, $4.2 \mathrm{mEq} / \mathrm{L}$; chloride, $105 \mathrm{mEq} / \mathrm{L} ; \mathrm{HCO}_{3}$, $24 \mathrm{mEq} / \mathrm{L}$.

The results of arterial blood gases performed with the patient breathing room air are as follows: $\mathrm{pH}, 7.38$; $\mathrm{pCO}_{2}, 40 \mathrm{pO}_{2}, 82 \mathrm{O}_{2}$ saturation, 94\%. The electrocardiograph (ECG) showed a sinus rhythm with a rate of 78/minute. The PR interval was 0.16 , QRS duration was 0.08 , and the axis was $60^{\circ}$. There were no ST-T wave segment abnormalities suggesting ischemia or infaction. 
A chest radiograph demonstrated minimally flattened diaphragms with mild hyperinflation of the lung fields consistent with chronic obstructive airway disease. There were no infiltrates or effusions and the cardiac silhouette was of normal size.

The diagnosis of cough syncope complicating an acute purulent bronchitis was made and erythromcin stearate, $250 \mathrm{mg}$ po, four times a day for 10 days was prescribed. The patient was instructed on the use of an over-the-counter cough suppressant and acetaminophen for fevers. Bedrest was advised. The patient returned home.

Approximately 8 hours after leaving the emergency department, the patient had a prolonged 1-hour episode of continuous chest pain with associated breathlessness. She refused to have her family call the paramedics because "the doctor said my heart was fine." One half hour later, the family found her unresponsive. When the paramedics arrived, the patient was in asystole. The Advanced Cardiac Life Support treatment protocol was administered without effect. The patient was transported to our emergency department and declared dead on arrival.

Dr Michael Kobernick: How did you find out about this?

Dr Howes: The following day, the attending physician who had pronounced the patient dead was kind enough to wait until I had finished my clinical shift before gently breaking the news to me. She specifically commented on the concise, complete nature of my documentation and that the discharge planning and diagnosis were appropriate to the history and physical examination findings noted. The physician mentioned that this could have very well have happened to any of us. Dr Kobernick: How did you feel?

Dr Howes: I can best answer that question by briefly outlining an experience $I$ had 9 years ago, several months after I began practicing emergency medicine. I saw a patient who was obviously ill and, despite the concerns of myself, another physician, nursing staff, and family members, this patient refused proper medical evaluation and left the hospital against medical advice. My worst fears were confirmed by her subsequent death. I was angry at the patient as well as myself. My confidence as a physician controlling the destiny of patients for whom I gave care was devastated. My rumination as to what I or others should have done differently was endlessly frustrating. My integrity as a physician was severely threatened. I became depressed.

I was fortunate to have a tremendous amount of support from several resident physicians, the emergency department director, and the nursing staff; there

\$The Emergency Medicine Residents, University of Illinois, Emergency Medicine Residency, Chicago.

were no intimations of misconduct on my part. The lack of malice on the part of the deceased family was especially helpful.

This reflection on events of the distant past are relevant today. When I heard of the outcome in the current case, the feelings I experienced were immediate, an amnestic response from the past. The mortality was the patient's, the morbidity was mine. I share this with you because we all have or will experience similar feelings.

Dr Kobernick: The stress of the death of a recently seen patient is common to all physicians. The fear of missing something is of great concern to the emergency physician with our limited encounters with patients. There are many stresses unique to physicians and some that are particular to emergency physicians. The focus of this session is on those that are unique and make our job more difficult than other specialties. University of Illinois ( $U$ of I) Residents: $\S$ The group discussion generated a list of stressors (Table 1) which included: "life in a fishbowl," shift changes, irregular schedule, "wrath of the medical staff," persistent treatment as an intern, lack of job security, normative crisis in the context of a highly stressful job, relationship to nursing staff, and pressure to keep the department flowing (disposition driven).

Dr Leslie Zun: According to a letter from John Pfifferling, $\mathrm{PhD}$, of the Center for the Well Being of Health Professionals, a similar problem list for emergency physicians has been generated. The problem list includes control over the ED, power, lifestyle, family, isolation, alienation, and poor colleague communica-

TABLE 1. List of Emergency Physicians' Stressors

Shift changes
Scheduling difficulties
Job insecurity
Pay reductions
Intrainstitutional visibility and scrutiny
Job satisfaction
Malpractice and litigation
Physician respect
Conflict with a patient's personal physician
Status of emergency physicians
Effects of diagnostic-related groups
Dealing with sudden death
Creating vegetative states
Threat of violence
Relationships with nursing, administration, and superiors
Fear of making a mistake
Difficulties of transferring patients
Back-up
Telemetry
Problem patients-abusive, demanding, ethanol abusers
Life decisions
Marital responsibilities
Problems associated with residents rotating on other services
Societal fears of hopelessness, crime, and poverty
Demands of professional responsibilities


tion. An informal survey performed by the American College of Emergency Physicians (ACEP) looked into the aspects of emergency medicine that are most stressful. Although the study was performed to identify trends, they found erratic schedules, unreasonable patient requests and expectations, fear of making a mistake, and threat of malpractice rated the highest. (Written Communication, November 1985)

Dr Kobernick: Working out of concert with the rest of the world starts out being a novelty. This soon wears off and the irregular schedule, mixed night and day shifts with the frequent changes rapidly lends to a burned out, cynical physician. The larger the emergency department, the more varied the schedule because of the greater variety of shifts. This phenomena seems to relate to the emotional changes an individual goes through in the transition from the 20 s to the $30 \mathrm{~s}$. In our 20s, we tend to be "footloose and fancy-free." The idea of working odd hours and having "lots of time off" appeals to this yearn for freedom. We are surprised when, in our $30 \mathrm{~s}$, we begin to search for some roots and regularity in our lives. This is where the stress of the schedule is most acutely felt. Often during this decade, there is a movement of emergency physicians to the more administrative positions with the more regular daytime schedule.

Dr Zun: Michaels wrote a review on the deleterious efforts of night-shift work in relation to the emergency physician. The major consequence of this type of changing work pattern is the disruption of social activities and sleep patterns. He recommends working single night shifts, proper daytime sleep environments, creating restful surroundings, duplicating physiologic eating schedules, and scheduling meetings and activities as a convenience. ${ }^{1}$

Dr Kobernick: The "wrath of the staff' refers to constant hostility many of us receive from members of our medical staffs when we interact with them. In a large number of institutions, emergency physicians are perceived as medical misfits; the physicians that "couldn't make it in practice." We are resented because we have no office overhead, no beeper to carry, and excessive time off. This false perception is used by our colleagues to justify their treatment of us. It is the rare physician who understands the difficulty of our job and gives us the credit we deserve. There are times when it creates considerable anxiety to call one of these attendings. This is unique to our field and we need to give ourselves credit for carrying this burden. Dr Zun: Anzinger, in his review of the emergency physician, "Man in the Middle," discussed some of the problems of working with the medical staff. He states, "An emergency physician is an unsolicited consultant who may be seen as an interloper and may even be viewed as disruptive to the physician-patient relationship" and ". . . (that) other medical staff mem- bers feel they need not worry about their relationship with him."2 Some medical staff view the emergency physician as a general practitioner who need not be listened to by the specialist, unwilling or unable to be responsible for ongoing medical care. If this situation does not stress the emergency physician enough, he is also called upon to enforce hospital bylaws that the medical staff finds troublesome. ${ }^{2}$ An example of this is calling another physician when the on-call physician does not respond by the prescribed time.

Dr Kobernick: The majority of the first 2 years of the residency are spent rotating on other services, and emergency medicine residents feel like outsiders and are treated as such. They are frequently relegated to the "scut" work that the medical student was not available to perform. In many ways, this is similar to the "wrath of the medical staff" mentioned above, and might represent training for the practice of emergency medicine.

Although not completely unique to individuals in emergency medicine, it is important to discuss the normative crises in the context of the emergency physician. Life transitions and crisis, such as marriage, divorce, child-birth, and death of a family member, are events that occur in everyone's life. They are more difficult to handle when one has a highly stressful job. It can even be difficult to get time off to go to a funeral. One's colleagues are often reticent to fill in for someone who needs coverage. Aside from the scheduling problems this creates, the physician often has a difficult time putting the personal crisis aside to focus on the clinical demands of critically ill patients. Emergency physicians must be right $100 \%$ of the time when making decisions. At times of personal crisis, most emergency physicians face the additional burden of having to perform at a very high level. Our system fails to recognize that there are times when human needs make it difficult for us to perform at this level. I have seen physicians fired from positions because they were in a personal crisis that would have resolved in a few months given a supportive environment. We need to think and act creatively when faced with physicians undergoing normative crises.

$U$ of I Residents: We understand there are stresses unique to the emergency physician. How do they manifest themselves on our lives?

Dr Kobernick: The ability to be effective in the emergency department and enjoy one's life is dependent upon keeping the stress in balance with the other aspects of life. When something comes out of balance, the scales are tipped and something suffers. If the job suffers, we make a fatal error, we have no leeway. The job may suffer in other ways that need attention before a crisis develops. Physicians who are suffering are often late for work, disinterested, slower, and less complete than usual, and show a change in their estab- 
lished work habits. This is a cry for help. Left to fester, these symptoms can lead to full-blown impairment with attendant substance abuse, suicide, depression, and other psychiatric illnesses.

Dr Zun: It is unknown how many emergency physicians are leaving emergency medicine for some other practice or have some form of impairment. The only inference to the magnitude of this problem is a review of impaired physicians in treatment in Georgia. Talbolt et al found that emergency medicine had the third highest impaired physician rate, behind anesthesia and general practice. ${ }^{3}$

Resident: What can we do in our own lives to lessen the effects of this difficult job?

Dr Kobernick: The first thing to do is for all of us to realize and give ourselves credit for doing something very difficult. The awareness leads to discussion, which is the next thing to do. We need to talk to each other about the stress and other facets of our lives that are positive or negative. We need to care for each other and think less about ourselves. Awareness, discussion, and concern for each other are the strongest weapons in the battle against the effects of stress.

Dr Zun: Physicians are reluctant to discuss their psychological discomforts. McCue states that "open direct exploration of productive adaptations to stresses would help to avoid suppression of feeling or excessive emotional distance from patients; such approaches would ultimately result in better, more economical medical care." 4

Stress is the emotional and physical response of the body to any demand made upon it. ${ }^{5}$ People have different reactions to stress. Appropriate coping mechanisms for stress may actually improve the quality of medical care, whereas those with inappropriate reactions or those with vulnerabilities may become impaired. ${ }^{6}$ There are no good studies of the stressors, effects of stress, coping mechanisms, or amount of impairment of physicians in emergency medicine. However, there is material on the effects of stress on other health care professionals in emergency medicine.

Studies of stress can be found in the nursing and paramedic literature. Burns, Keriloff, and Close studied the sources of stress and satisfaction in emergency nursing. They found inadequate staffing and working with an apathetic, inexperienced physician to be the greatest sources of stress. ${ }^{7}$ Mytych found that emergency medicine nurses have a moderate degree of burnout. She also studied certain factors of job satisfaction and burnout and in 40 ED nurses; burnout levels correlated with job performance, promotion, and supervision, but no correlation was found between pay and coworker relationships and burnout. ${ }^{8} \mathrm{~A}$ recent study of paramedics found a high degree of occupational stress compared with other medical personnel. ${ }^{9}$
McSwain and Skelton have commented on solutions to the almost $40 \%$ burnout rate of trained emergency medical technicians. ${ }^{10}$

Although much is written in the medical literature concerning stress, impairment, and burnout of the physician, none specifically addresses the emergency physician. Mawardi studied the source of stress for the graduates of Case Western Reserve School of Medicine. He found time pressures, therapy failure and patient-related problems, and office details and paperwork to rate among the top stressors. ${ }^{\text {" }}$ Krahowski found the most important sources of stress were dealing with noncompliant or recalitrant patients, nurses neglecting duties, and excessive paperwork. He also commented on the severity of stress and its effects on physicians. ${ }^{12}$ Some physicians have written about methods to counteract these stressors. Spears has presented a time management system to prevent impairment. ${ }^{13}$ Gruze has written a commentary on forestalling professional dissatisfactions and regaining an enthusiastic approach to medicine. ${ }^{14}$

Most emergency physicians agree that emergency medicine is a stressful field, but only one study has attempted to measure the level. Lehman et al measured the urine catecholamines and stress index of paramedics and five physicians involved in emergency care in Germany. The investigators examined reactions to routine cases and compared them with reactions while caring for seriously injured patients. They found that the physicians had a higher level of urine adrenaline excretion and blood lactate level than the paramedics. They theorized that this may be the consequence of the overall responsibilities. Because of this burden, Lehman et al believe that coronary insufficiency or hypertension may be contraindications for emergency medical service. ${ }^{15}$

There are many studies of the stress reaction of health care workers involved in mass casualty care. These include role conflicts, feelings of frustration and futility, loss of confidence, grief reactions, "burnout," and stress. ${ }^{16-19}$ Sanner and Wolcott studied the stress reactions of the participants in five large military casualty simulations. He found that the participants, including emergency physicians, had not only consistent stress reactions but also reactions that were similar to those of other health care workers. ${ }^{19}$

Physicians who work in the critical care setting have specific psychological reactions to acute medical illness. Strain believes that physicians are vulnerable to the same stresses as their critically ill patients. Although physicians are not sufficiently aware of their stress, these stressors parallel those that they were exposed to in their early psychological development. For example, the patient who doesn't get better may elicit in the physician a loss of narcissistic integrity. Strain states that "the physician must also possess 
sufficient self-knowledge to recognize and cope with the stresses under which he himself is operating." $\mathrm{He}$ goes on to emphasize that critical care specialists must identify their levels of tolerance for dealing with the dying patient and the feelings involved. Physicians must accept this as human limitation rather than personal inadcquacy. ${ }^{20}$

There are many techniques to reduce stress. In 1985, ACEP polled some of its membership to determine techniques or approaches used to reduce stress load. They found that maintaining a positive attitude about work, exercise/sports, vacations and time off, and a healthy lifestyle rated the highest. (Written Communications, November 1985) Everly and Rosenfeld, in their text on stress response for the clinician, discuss many of these techniques: dietary recommendations, pharmacological treatment, meditation, neuromuscular relaxation, voluntary control of respiratory pattern, biofeedback, physical exercise, and hypnosis. ${ }^{5}$ Benson, Beary, and Carol studied the effects of the relaxation response. They commented on the value of the relaxation response in situations of excessive sympathetic activity. ${ }^{21}$ Other techniques include lifestyle modifications, changes in the workplace, communication with colleagues, and leisure diversions.

The aforementioned techniques may be valuable activities during time off but do not assist physicians who are under stress in the treatment area. Some physicians recommend taking a "minute out" in a quiet area, wearing comfortable clothes, or making time to have a meal. Thompson and colleagues surveyed attending and resident emergency physicians to develop a list of stress management techniques. This list includes understanding limitations, frequent use of humor, and fostering department teamwork. ${ }^{22}$ An emergency physician needs to find a technique that is effective as a stress reducer and use it.

In conclusion, the emergency physician is exposed to many stressors, some of which are yet to be identified. We suspect that these may, without adequate coping mechanisms, lead to physician impairment and "burnout." Further study of the stressors and their effects on emergency physicians is needed to prevent impairment of the specialty.

\section{REFERENCES}

1. Michaels HE. Night shift work. Ann Emerg Med 1984; 13:201-202
2. Anzinger RK. Emergency department physician: Man in the middle. Hospital Medical Staff 1976;5:10-13

3. Talbott GO, Grallegos KV. Wilson PO, et al. The medical association of Georgia's impaired physician program. Review of the first 1,000 physicians: Analysis of specialty. JAMA 1987;257:2927-2930

4. McCue JD. The effects of stress on physicians and their medical practice. N Engl J Med 1982;306:458-463

5. Everly GS, Rosenfeld R. The Nature and Treatment of the Stress Response: A Practical Guide for Clinicians. New York, Plenum, 1981;3-11, 71-173

6. McCue JD. The effects of stress on physicians and their medical practice. N Engl J Med 1982;306:458-463

7. Burns HK, Kirolloff LH, Close JM. Sources of stress and satisfaction in emergency nursing. J Emerg Nurs 1983; 9:329-336

8. Mytych KM. Burnout in the emergency department nurse. $J$ Emerg Nurs 1981;7:265-268

9. Hammer JS, Matthews JI, Lyon JS, et al. Occupational stress within the paramedic profession. Ann Emerg Med 1986;15:536-539

10. McSwain NE, Skelton MB. Burnout-Real or imagined. Top Emerg Med 1980;1:93-97

11. Mawardi $\mathrm{BH}$. Satisfaction, dissatisfactions, and causes of stress in medical practice. JAMA 1979;241:1483

12. Krakowski AJ. Stress and the practice of medicine. Stress stresses, and strains. Psychother Psychosom 1982; $38: 1 \uparrow-23$

13. Spears BW. A time management system for preventing physician impairment. J Fam Prac 1981;13:75-80

14. Gruze SB. Can the practice of medicine be fun for a lifetime? JAMA 1979;241:2021-2023

15. Lehmonn, Dorges $V$, Huger $G$, et al. Zum Verhalten der fuen Katecholamine im Blut and Hain bei Sanisatein and Arzenten Wahrend des Einsatzes (Abstr) Int Arch Occup Environ Health 1983;51:209-222

16. Raphael B, Singh B, Bradbury L. Disaster: The helper's perspective. Med J Aust 1980;2:445-447

17. Hartman $K$, Allison J. Expected psychological reactions to disaster in medical rescue teams. Milit Med 1981; 146:323-327

18. Durham TW, McCammon SL, Allison EJ. The psychological impact of disaster on rescue personnel. Ann Emerg Med 1985;14:664-668

19. Sanner PJ, Wolcott BW. Stress reactions among participants in mass casualty simulations. Ann Emerg Med $1983 ; 12: 426-428$

20. Strain JJ. Psychological reactions to acute medical illness and critical care. Crit Care Med 1978;6:39-44

21. Benson $H$, Beary JF, Carol MP. The relaxation response. Psychiatry 1974;37:37-46

22. Thompson BM, Smereka $C$, Moskal W, et al. Evaluating clinical supervision in an emergency medicine program: Resident and staff perception of effective supervision and stress management (Abstr) Ann Emerg Med 1987;16:503 